\title{
Ratio scales of sugar sweetness
}

HOWARD R. MOSKOWITZ2
HARVARD UNIVERSITY

In a series of 10 experiments, groups of Os judged the sweetness of 16 sugars. The results suggest that, for all sugars except mannose, the intensity of sweetness grows as a power function of concentration, with an exponent of about 1.3. The relative sweetness of sugars was determined using both molarity and per cent by weight. With both measures, sucrose and fructose were the sweetest sugars. The order of the remaining sugars in the sweetness hierarchy was partly a function of the measure of concentration. The variability of the magnitude estimates of sweetness was roughly proportional to the stimulus concentration, supporting Weber's law.

The sweetness of sugars can be scaled along two major dimensions: quality and intensity. Little work appears to have been done to quantify the differences in quality among sugars, other than a listing of the more obvious sensory effects. For example, the sweetness of glucose differs markedly from that of sucrose, with a burning side taste often appearing at high concentrations (Amerine, Pangborn, \& Roessler, 1965). Mannose, a monosaccharide similar in structure to glucose, tastes both bitter and sweet (Pangborn \& Chrisp, 1966; Pangborn \& Gee, 1961).

Differences in sweetness intensity have been much explored. Sugars differ in their sweetening power, forming a hierarchy, with sucrose and fructose at the apex and lactose and raffinose at the base (Cameron, 1947). Complications in the hierarchy often arise because of the presence of anomers having similar chemical properties but differing from each other primarily in their ability to rotate light. The gustatory response is different to each anomer, so that at equal concentrations, the two forms differ in sweetness, e.g., $\alpha$ glucose is sweeter than $\beta$ glucose, whereas $\beta$ lactose is sweeter than its anomer, $\alpha$ lactose (Pangborn \& Chrisp, 1966; Pangborn \& Gee, 1961).

Traditionally, relative sweetness has been defined as the ratio of concentrations of substances matching in sweetness. For example, if $8 \%$ maltose and $4 \%$ glucose taste as sweet as $2 \%$ sucrose, then maltose is $25 \%$ as sweet as sucrose, whereas glucose is $50 \%$ as sweet. The tacit assumption made is that the ratio of physical concentrations that match defines a like ratio of sensory intensities. The sensory ratio is not directly measured, although it could be measured by asking $O s$ to estimate the relative magnitude of sweetness of sugars at the same concentration. This estimation procedure has proved effective in measuring sensory ratios.

The present study concerns the function relating sweetness to concentration of sugar and is designed to compare the parameters of the function across different sugars. The sensory judgments of sweetness were obtained by magnitude estimation, a method in which Os assign numbers to stimuli in proportion to the perceived sweetness. The results of many similar

Table

experiments on the growth of sensory intensity suggest that, for more than two dozen perceptual continua, a power function, $S=k I^{n}$, relates sensory intensity, $S$, to physical intensity, I. In $\log \log$ coordinates, the power function becomes a line, $\log S=n \log I+\log k$, with slope $n$ and intercept $k$. Much attention has been directed to the exponent (slope $n$ ) of each continuum because it is a relatively invariant parameter across different experiments. The exponent characterizes the transformation of stimulus ratios into sensory ratios and is independent of the absolute physical intensities of the stimulus and of the modulus of the scale chosen by

Stimulus Concentrations

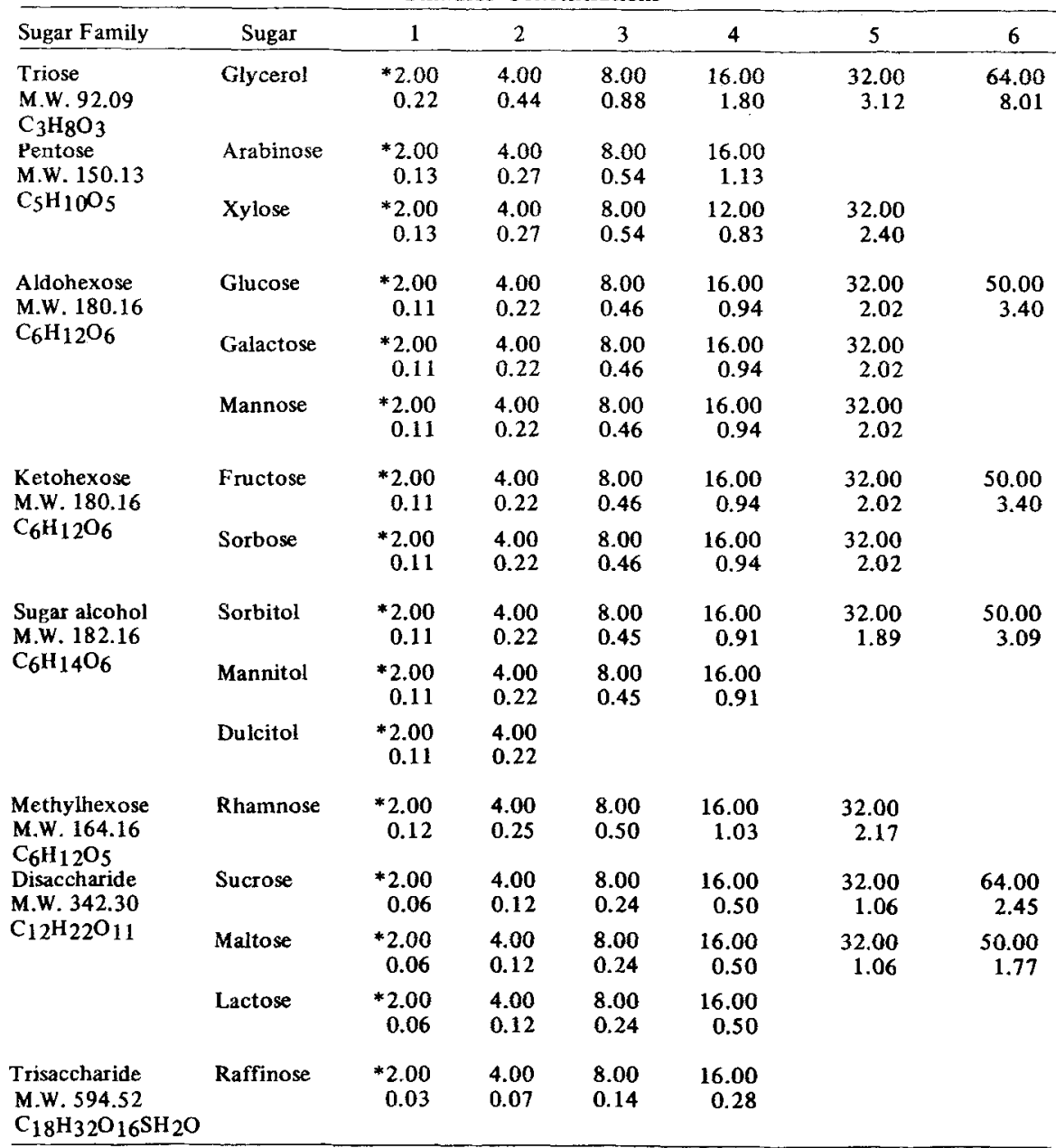

* Asterisks indicate that concentration is expressed as per cent by weight; non-asterisked rows indicate that concentration is expressed as molarity. 
Table 2

Conditions of Experiment

\begin{tabular}{|c|c|c|c|}
\hline $\begin{array}{l}\text { Experi- } \\
\text { ment }\end{array}$ & $\begin{array}{c}\text { No. } \\
\text { of } \\
\text { Observers }\end{array}$ & $\begin{array}{c}\text { Judgments } \\
\text { per } \\
\text { Observer } \\
\end{array}$ & Stimuli \\
\hline 1 & 40 & 1 & $\begin{array}{l}\text { Fructose, Galactose, Glucose, Lactose, Maltose, Mannitol, } \\
\text { Sorbitol, Sucrose }\end{array}$ \\
\hline 2 & 10 & 2 & Maltose, Mannose, Raffinose, Sorbose, Sucrose \\
\hline 3 & 10 & 2 & Arabinose, Mannose, Raffinose, Rhamnose, Sucrose \\
\hline 4 & 10 & 2 & Dulcitol, Galactose, Mannitol, Sorbitol, Sucrose \\
\hline 5 & 10 & 2 & Lactose, Raffinose, Sorbose, Sucrose, Xylose \\
\hline 6 & 10 & 2 & Fructose, Glucose, Glycerol, Sucrose \\
\hline 7 & 20 & 2 & Sucrose, Na Cyclamate*, Ca Cyclamate*, Na Saccharin* \\
\hline 8 & 20 & 2 & Sucrose, Artificial Sweetening Compounds* \\
\hline 9 & 30 & 1 & Glycerol, Sucrose, Artificial Sweetening Compounds* \\
\hline 10 & 30 & 1 & $\begin{array}{l}\text { Sucrose, Na Saccharin*, Sucrose and } \mathrm{Na} \text { Saccharin under } \\
\text { different levels of solution viscosity* }\end{array}$ \\
\hline
\end{tabular}

- Results nat reported here

the 0 . The intercept $k$ is the scale factor and may change from experiment to experiment without affecting the exponent (Stevens, 1960).

The exponent for sugar sweetness appears to be about 1.3. Stevens reported an exponent of 1.3 for both sucrose and glucose, and that value for sucrose was confirmed by magnitude estimation and cross-modal matching (with loudness) by Moskowitz (1968). On the other hand, Gregson and Russell (1965) reported an exponent of about 0.6 for the sweetness of sucrose. A discussion of their results and of others' appears in Moskowitz (1968).

When the relative sensory intensities of different sugars are of interest, the intercept also becomes important, since it gives the relative sensory intensity of different compounds at one point on the concentration continuum. At the concentration given by the intercept, the sweetness of different sugars may be easily compared. Meaningful intercept may be ensured by presenting different sugars thereby allowing the $O$ to scale them relative to a common unit. That was done in the present study by using a core set of six sucrose concentrations in every experiment.

\section{STIMULI AND PROCEDURE}

In 10 experiments, different groups of Os from a pool of 83 judged the sweetness of 16 sugars (Tables 1 and 2) by magnitude estimation. All solutions except sucrose were made from reagent-grade chemicals during the same experimental session,

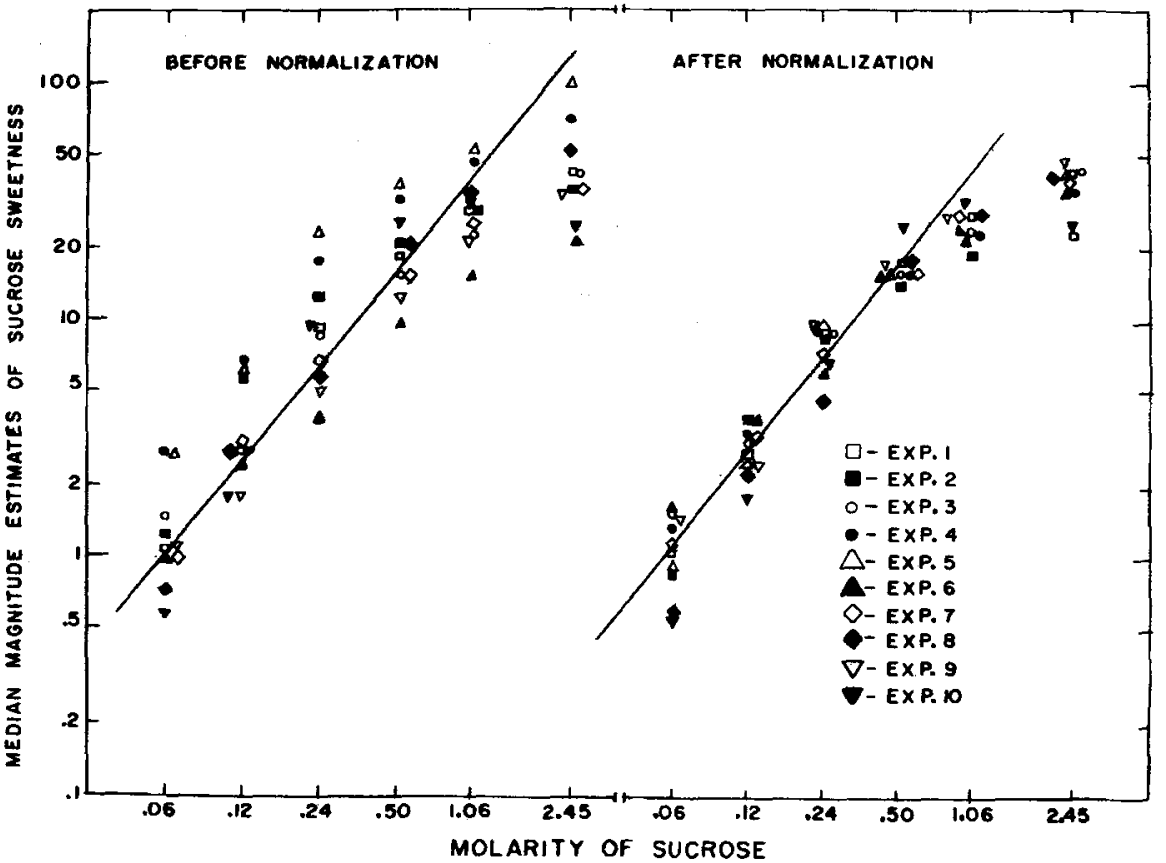

and Cambridge tap water. Sucrose was obtained from a commercial brand of cane sugar (Domino). Solutions were stored at $5^{\circ} \mathrm{C}$ and were sampled at room temperature $\left(19^{\circ} \mathrm{C}\right)$. Prior to use, solutions remained under refrigeration for 3 days in order to ensure mutarotation to an equilibrium mixture of anomers.

During the experimental session, the stimulus solutions were presented to the $\mathrm{O}$ in paper cups, with 5 to $10 \mathrm{ml}$ of solution in each. The solutions were given in an irregular order to the $O$, who first sampled the solution, then gave a magnitude estimate of sweetness, and finally rinsed with tap water. There were no restrictions on time, so that the $O$ completed the session as quickly as he wished (usually in $20 \mathrm{~min}$ ). He was given the following written instructions:

"In front of you is a series of paper cups filled with stimulus solutions. Your task is to tell how sweet they seem by assigning numbers proportional to sweetness. If the second stimulus is nineteen times as sweet as the first, assign it a number nineteen times as large. If it seems one-eleventh as sweet, assign it a number one-eleventh as large, and so forth. Use numbers, fractions, and decimals, but make each assignment proportional to the sweetness as you perceive it."

\section{ANALYSIS OF THE MAGNITUDE ESTIMATES}

Since magnitude estimates are usually distributed log-normally (J. C. Stevens, 1957), the geometric mean is usually a proper measure of central tendency. In the present study, however, it was often inapplicable, since many Os gave magnitude estimates of " 0 " when they could not detect sweetness. In this situation, the median is the preferred measured. For computer analysis, however, judgments of " 0 " were replaced by a number equal to 0.1 times the lowest magnitude estimate given by the $O$. The medians and the geometric means of the new distributions tended to agree with each other, suggesting that the foregoing replacement procedure did not severely distort the original distribution of magnitude estimates. A computer program called Psychofit (Panek \& Stevens, 1965)

Fig. 1. Median magnitude estimates of sucrose sweetness, before and after the results of 10 experiments were brought into line with each other by normalizing. The line drawn through the four lowest concentrations has been given a slope of 1.3. The coordinates are log-log, suggesting that sucrose sweetness conforms to a power function of concentration, with an exponent of 1.3 . 
Table 3

Parameters of the Power Functions for Sugars $\left(S=\mathrm{k}^{\mathrm{n}}\right.$ ). The parameters were obtained for two cases: the exponent was fixed at 1.3 , or the exponent was not fixed.

\begin{tabular}{|c|c|c|c|c|c|c|c|c|c|c|c|c|}
\hline & \multicolumn{6}{|c|}{ Molarity } & \multicolumn{6}{|c|}{ Per Cent by Weight } \\
\hline & \multicolumn{3}{|c|}{$\mathrm{n}=1.3$} & \multicolumn{3}{|c|}{ Least-Squares Fit } & \multicolumn{3}{|c|}{$\mathrm{n}=1.3$} & \multicolumn{3}{|c|}{ Least-Squares Fit } \\
\hline & Rank & $\mathbf{k}$ & R.S.* & $\mathbf{n}$ & $\mathbf{k}$ & R.S.* & Rank & $\mathbf{k}$ & R.S.* & $\mathbf{n}$ & $\mathbf{k}$ & R.S.* \\
\hline Sucrose** & 1 & 50.0 & 1.00 & 1.33 & 46.4 & 1.00 & 1 & 0.54 & 1.00 & 1.35 & 0.43 & 1.00 \\
\hline Fructose** & 2 & 20.4 & 0.41 & 1.33 & 21.2 & 0.46 & 2 & 0.49 & 0.91 & 1.38 & 0.43 & 1.00 \\
\hline Raffinose & 3 & 16.6 & 0.33 & 0.63 & 3.5 & 0.07 & $1 \overline{5}$ & 0.08 & 0.15 & 0.64 & 0.27 & 0.63 \\
\hline Maltose & 4 & 15.0 & 0.30 & 1.20 & 13.4 & 0.28 & 12 & 0.17 & 0.31 & 1.25 & 0.19 & 0.44 \\
\hline Lactose & 5 & 11.0 & 0.22 & 0.89 & 5.5 & 0.12 & 14 & 0.12 & 0.22 & 0.98 & 0.23 & 0.53 \\
\hline Dulcitol & 6 & 10.8 & 0.22 & 0.24 & 1.5 & 0.03 & 5 & 0.25 & 0.46 & 0.24 & 0.74 & 1.72 \\
\hline Glucose & 7 & 9.4 & 0.19 & 1.18 & 8.9 & 0.19 & 4 & 0.24 & 0.45 & 1.26 & 0.22 & 0.50 \\
\hline Galactose & 8 & 9.2 & 0.18 & 1.36 & 9.6 & 0.21 & 6 & 0.23 & 0.42 & 1.43 & 0.17 & 0.40 \\
\hline Sorbose & 9 & 9.1 & 0.18 & 1.12 & 7.9 & 0.17 & 7 & 0.22 & 0.41 & 1.17 & 0.19 & 0.44 \\
\hline Sorbitol & 10 & 8.4 & 0.17 & 1.22 & 8.1 & 0.17 & 9 & 0.20 & 0.37 & 1.17 & 0.29 & 0.68 \\
\hline Mannitol & 11 & 7.7 & 0.15 & 1.24 & 7.7 & 0.16 & 11 & 0.18 & 0.33 & 1.27 & 0.19 & 0.44 \\
\hline Arabinose & 12 & 6.9 & 0.14 & 1.16 & 6.0 & 0.13 & 8 & 0.21 & 0.39 & 1.20 & 0.25 & 0.58 \\
\hline Rhamnose & 13 & 6.7 & 0.14 & 1.29 & 6.8 & 0.15 & 10 & 0.19 & 0.35 & 1.34 & 0.17 & 0.40 \\
\hline Glycerol & 14 & 5.2 & 0.10 & 1.09 & 4.7 & 0.10 & 3 & 0.27 & 0.50 & 1.13 & 0.40 & 0.93 \\
\hline Xylose** & 15 & 4.8 & 0.10 & 1.32 & 4.9 & 0.11 & 13 & 0.14 & 0.26 & 1.37 & 0.19 & 0.44 \\
\hline
\end{tabular}

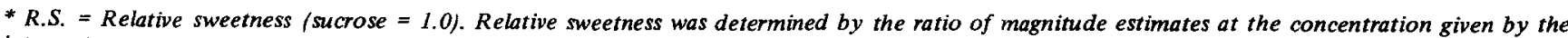
intercept.

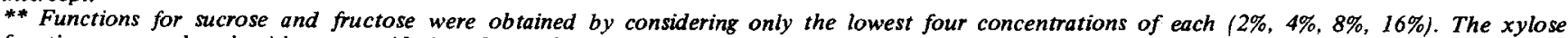
function was analyzed without considering the median magnitude estimate given to $2 \% x y l o s e$.

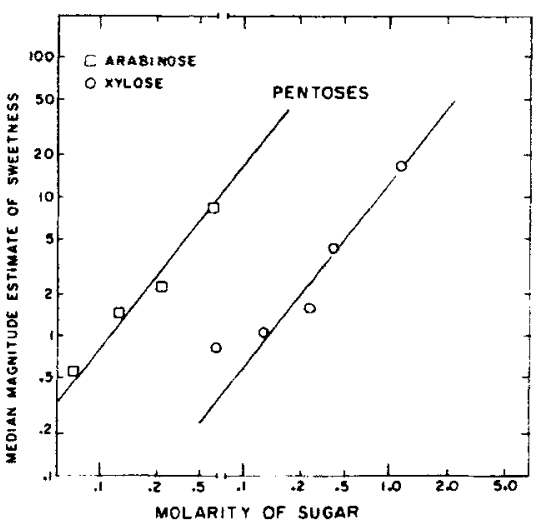

A

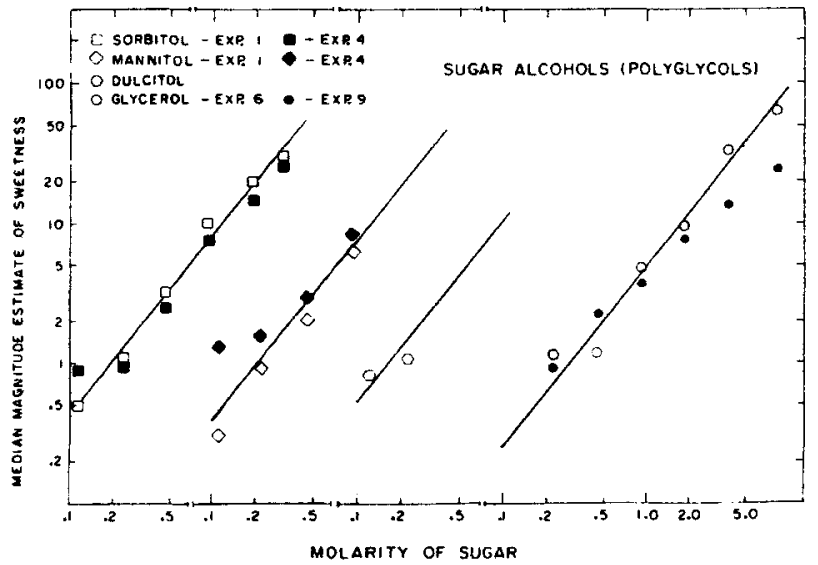

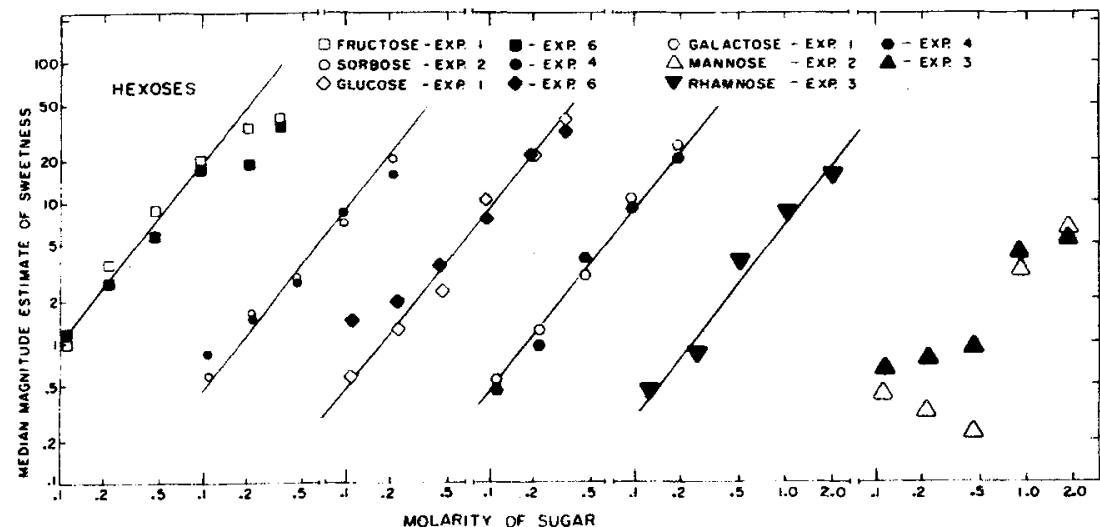

MOLARITY OF SUGAR

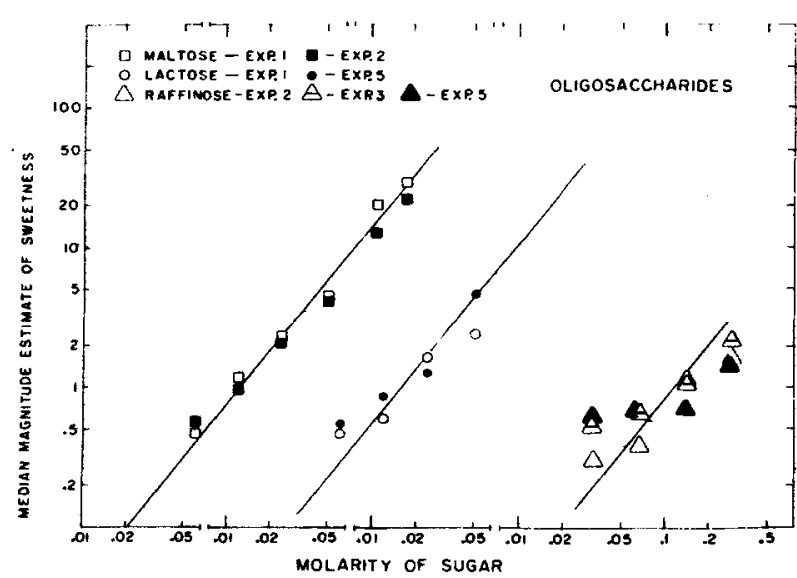

Fig. 2. Median magnitude estimates of the sweetness of (A) pentoses, (B) hexoses, (C) sugar alcohols (polyglycols), and (D) oligosaccharides (heavier sugars) after normalization. 
provided a least-squares fit of the exponent and intercept of the simple power function, $S=\mathrm{kI}^{\mathrm{n}}$, to the magnitude estimates. In addition, Psychofit provided summary statistics on the responses, including the variability of the judgments before and after the scale units used by different $O s$ had been equalized by modulus equalization (Lane, Catania, \& Stevens, 1961).

Since the absolute levels of the median judgments differed in each experiment, a normalization procedure was used to bring the magnitude estimates into agreement with each other. The geometric means of the magnitude estimates given for $4 \%, 8 \%$, $16 \%$, and $32 \%$ sucrose (Table 1) were multiplied by a number that made their grand mean come to 10.0 . The geometric means and medians for the other sugars scaled in the same experiment were also normalized by the same multiplier. A different multiplier was needed in each experiment. The normalization suppressed some of the intercept variability and allowed the slope of the sucrose function (Fig. 1) to emerge more clearly.

\section{RESULTS}

Form of the Sweetness Function

For 15 sugars (Figs. 1 and 2 and Table 3), the median magnitude estimate of sweetness grew as a power function of concentration. Lines drawn through the medians have slopes of 1.3 , a value chosen on the basis of previous estimates of the sweetness exponent (Moskowitz, 1968; Stevens, 1969). Although exceptions occur, a single representative exponent appears to be justified. For each sugar (except mannose), a least-squares fit was also obtained for the slope and intercept. The results (Table 3 ) suggest that, in most cases, the exponent departs only slightly from 1.3.

Several stimulus concentrations were not included in the foregoing analysis. Magnitude estimates given to $2 \%$ xylose $(0.13 \mathrm{M})$ depart from the rest of the xylose function and should not be blindly included in analyses by least squares. On the other hand, reproducible curvature at the top of the sucrose and fructose functions (Figs. 1 and 2B) suggest that systematic departures from the power function are occurring, so that these higher concentrations call for separate consideration.

Mannose (Fig. 2B) is the only sugar of the 16 that did not grow as a power function of concentration. The median magnitude estimates were erratic,

Fig. 3. Equal-sweetness functions traced out by matches between sucrose, glucose, fructose, and lactose. suggesting differences in the Os' perceptions at low concentrations. In one experiment, the sweetness of dilute mannose solutions decreased with increasing concentration, whereas in the other, it increased in the expected manner. The confusion of the sweet taste and the bitter side taste reported to accompany mannose may be implicated in this anomalous result.

Sweetness functions may be related to two measures of concentration, one using molarity and emphasizing the number of molecules in solution and the other using per cent by weight (grams solute per $100 \mathrm{~g}$ solution) and emphasizing the weight of solute in solution. The slopes and intercepts differ, depending upon the measure of concentration (Table 3). Per cent by weight constricts the range of the measure of concentration and always gives a slightly steeper slope (Moskowitz, 1968).

\section{Sweetness Functions from Other Studies}

The foregoing premise, that sweetness conforms to a power function of concentration, is supported by other data obtained by one or another matching method (Fig. 3). Matching functions with a slope of 1.0 (in log-log coordinates) suggest that both sugars have equal (but undetermined) exponents in the power law, whereas functions with slopes less than 1.0 indicate that the abscissa sugar has the lower exponent, i.e., grows less rapidly in sweetness. Glucose sweetness probably grows slightly more rapidly than sucrose sweetness (Functions 1-3, Fig. 3A), whereas the sweet taste of sucrose, lactose,
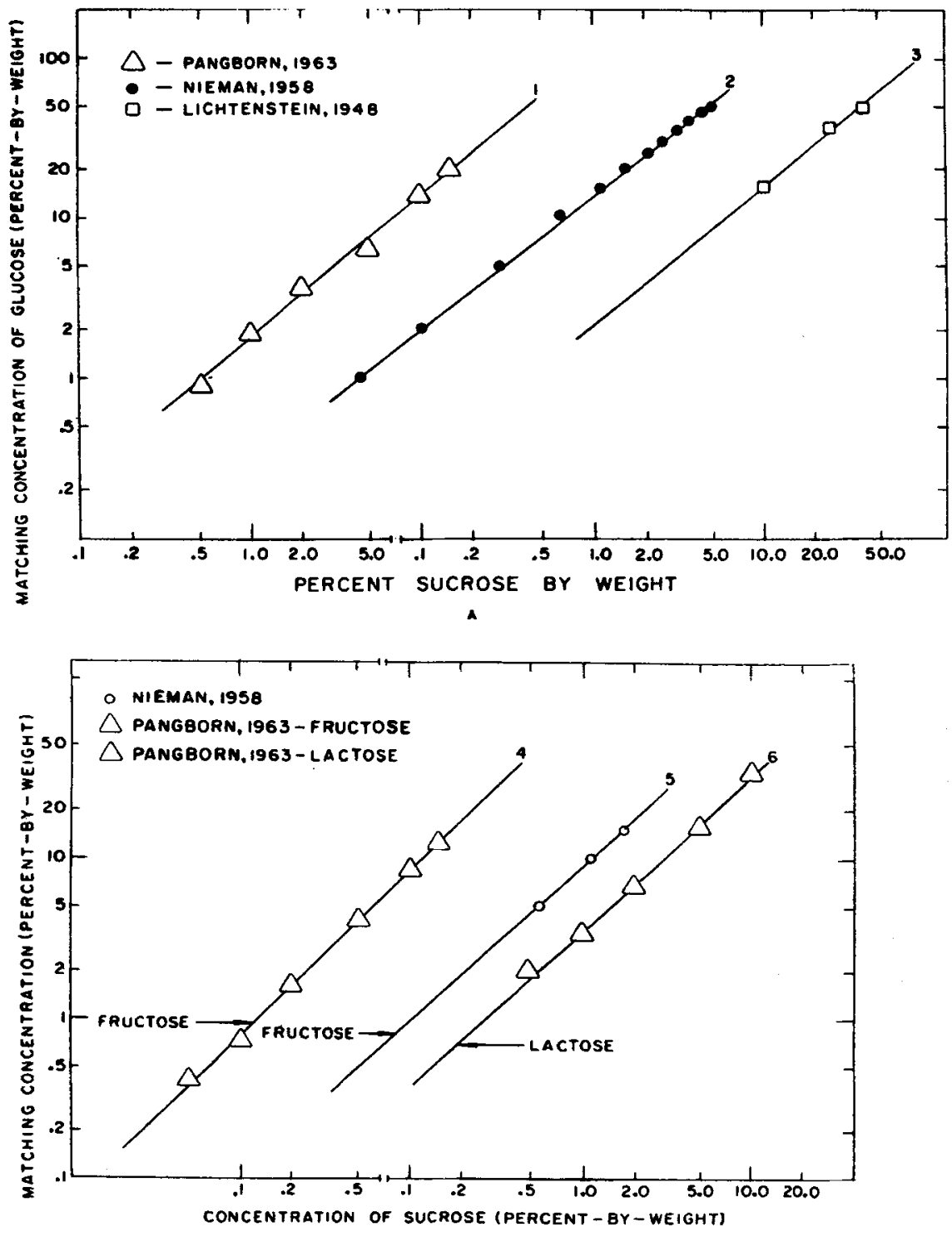

B 

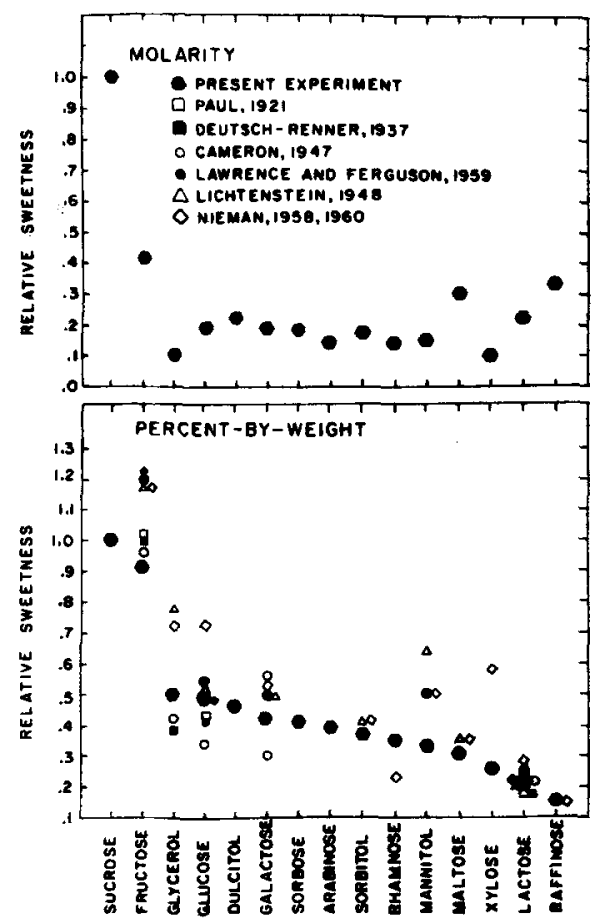

Fig. 4. Relative sweetness of sugars obtained in the present study and in previously published ones. In the present study, the values for relative sweetness were computed when concentration was expressed both as molarity. and as per cent by weight. The exponent for sweetness was fixed at 1.3. Estimates of relative sweetness attributed to Deutsch-Renner and Nieman were obtained from tabled values presented in Amerine, Pangborn, and Roessler (1965).

and fructose grow at about the same rate (Fig. 3B).

\section{Relative Sweetness of Sugars}

The ratio of magnitude estimates at each concentration expresses relative sweetness at that concentration. If the exponent for all sugar sweetness is 1.3 , then the ratio of intercepts (values of $k$ ) between pairs of sugars expresses their relative sweetness both at the concentration given by the intercepts as well as across the entire continuum of concentration. Thus, if one exponent suffices, then relative sweetness remains constant, as Eq. 1 indicates:

$$
\begin{aligned}
& \text { Sugar A: Sweetness } S_{A}=k_{A} C^{1.3} \\
& \text { Sugar B: Sweetness } S_{B}=k_{B} C^{1.3}
\end{aligned}
$$

Relative sweetness is the ratio of magnitude estimates at one concentration:

$$
\mathrm{S}_{\mathrm{A}} / \mathrm{S}_{\mathrm{B}}=\mathrm{k}_{\mathrm{A}} \mathrm{C}^{1.3} / \mathrm{k}_{\mathrm{B}} \mathrm{C}^{1.3}=\mathrm{k}_{\mathrm{A}} / \mathrm{k}_{\mathrm{B}}
$$

With one representative exponent, the

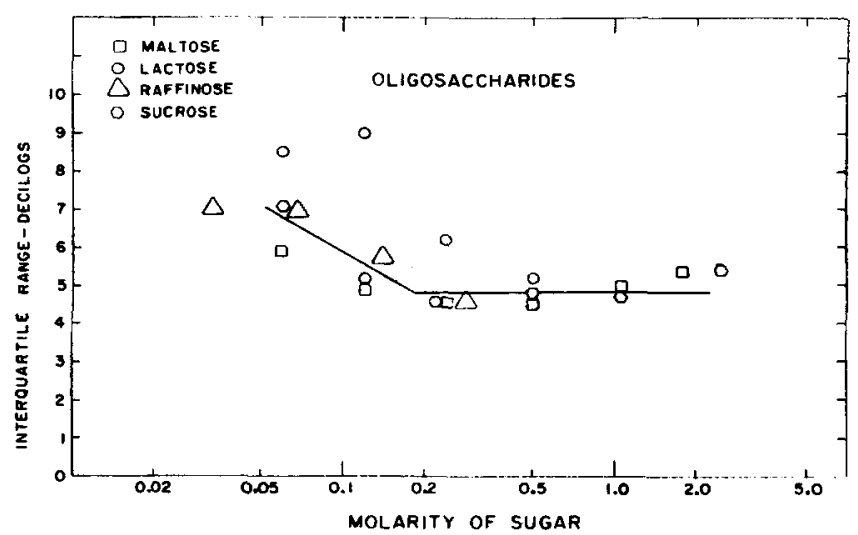

A

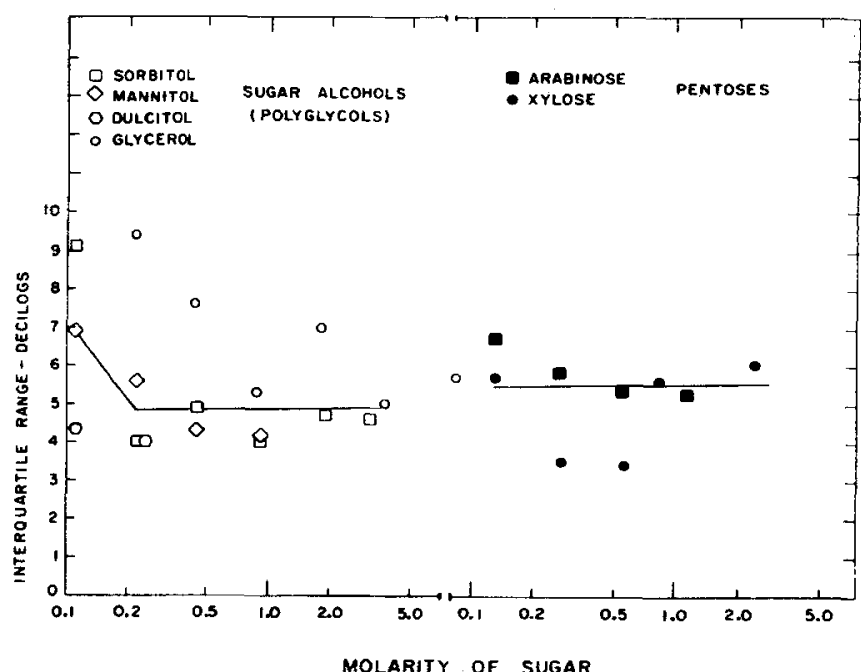

E

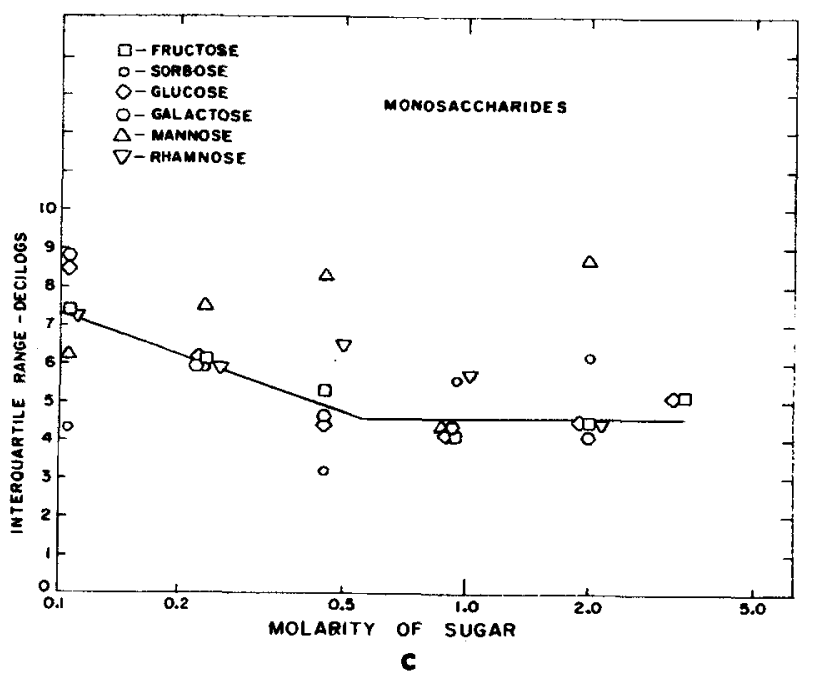

Fig. 5. Behavior of the interquartile range of magnitude estimates of the sweetness of (A) oligosaccharides, (B) pentoses and sugar alcohols, and (C) monosaccharides after modulus equalization was done to remove differences in the moduli used by different Os. The unit of variability is the decilog (10th of a logarithmic unit). 
sweetness functions become parallel lines in log $l o g$ coordinates, separated by a constant distance that corresponds to a fixed ratio. Table 3 lists the absolute and relative intercepts (sucrose $=1.0$ ), assuming the exponent to be 1.3. The intercepts were obtained by a least-squares fit, in which the exponent was fixed at 1.3.

Whether measured in terms of molarity or per cent by weight, sucrose and fructose are the sweetest sugars (Fig. 4), whereas for the remaining sugars, the measure of concentration influences the order of relative sweetness. When molarity is used, the heavier sugars (oligosaccharides), such as maltose and lactose, are sweeter than the lighter monosaccharides, whereas with per cent by weight, the order is reversed. This finding is intuitively meaningful, because molarity pits light sugars (e.g., glycerol) against much heavier ones (e.g., raffinose) without allowing for the differences in weight. On the other hand, per cent by weight assesses the amount of solute in solution, regardless of its molecular weight.

Values for dulcitol and raffinose are more tentative than are the rest, because neither sugar ever becomes sufficiently sweet to determine a good sweetness function with many points.

\section{Relative Sweetness from Other Studies}

The results of other studies were analyzed to determine relative sweetness. The pairs of concentrations of sugars matching in sweetness were substituted in Eq. 1; the two parts were set equal, and the equation was solved for the ratio of intercepts (under the assumption that the sweetness exponent was 1.3). There is a spread of values (Fig. 4) for the relative sweetness of sugars, especially for glucose. Some of the variability can be traced to different procedures used by the different Es, but the remainder may be attributable to differences of the sweetness exponents of different sugars. If, for example, the sweetness exponent of glucose exceeds that of sucrose, then their relative magnitude estimates will differ across concentrations. Rather than one representative value, there will be an entire distribution (Eq. 3):
Sugar A: Sweetness $S_{A}=k_{A} C^{m}$

Sugar B: Sweetness $S_{B}=k_{B} C^{n} \quad n \neq m$ (3)

Relative Sweetness $=S_{A} / S_{B}=1, C^{m} / k_{B}$ 1337-1344. ( Society of America, 1960, 32, AWRENCE, A. R., \& FERGUSON, L. N. Exploratory physiochemical studies on the sense of taste. Nature, 1959, 183, 1469-1471.

$$
=\mathrm{k}_{\mathrm{A}} / \mathrm{k}_{\mathrm{B}} \times \mathrm{C}^{\mathrm{m}-\mathrm{n}}
$$

LICHTENSTEIN, P. E. The relative sweetness of sugars: Sucrose and dextrose. Joumal of Experimental Psychology, 1948, 38, 578-580.

Instead of traveling in parallel paths, separated by a constant distance, the functions for pairs of sugars cross, so that the logarithmic distance (i.e., ratio) continually changes with concentration.

\section{Variability of Magnitude Estimates}

Figure 5 shows how one measure of variability, the interquartile range, behaves for different sugars. The statistic was computed from the logarithms of the magnitude estimates after the scale sizes were equated by modulus equalization. Each point is a weighted value, obtained from several experiments, with weights proportional to the number of judgments obtained in each experiment. In general, the variability behaves in a familiar pattern. At low concentrations, the interquartile range is relatively high, but it stabilizes over the top $80 \%$ of the stimulus continuum. A similar pattern occurs for the variability of matches of numbers and noise to the intensity of taste and of taste mixtures (Moskowitz, 1968).

If the interquartile range of the judgments (in logarithmic units called decilogs) is constant, then the variability of the judgments is proportional to the mean judgment. This stability of relative variability reaffirms Weber's law, which posits that the variability of sensory judgments is relative and increases with increasing intensity.

\section{REFERENCES}

AMERINE, M., PANGBORN, R., \& ROESSLER, E. Principles of sensory evaluation of food. New York: Academic Press, 1965.

CAMERON, A. T. The taste sense and the relative sweetness of sugar and other sweet substances. Scientific Reports of the Sugar Research Foundation, No. 9, New York, 1947. sucrose intensity. Perceptual \& Motor Skills, 1965, 20, 294.
MOSKOWITZ, H. R. Scales of intensity for single and compound tastes. Unpublished doctoral dissertation, Harvard University, 1968.

PANEK, D. W., \& STEVENS, J. C. Psychofit, a computer program for the treatment of psychophysical data. Laboratory of Psychophysics, Harvard University, 1965. PPR 315.

PANGBORN, R. M. Relative taste intensities of selected sugars and organic acids. Journal of Food Science, 1963, 28, 726-733.

PANGBORN, R. M., \& CHRISP, R. B. Gustatory responses to anomeric sugars. Experientia, $1966,22,612-615$.

PANGBORN, R. M., \& GEE, S. Relative sweetness of $\alpha$ and $\beta$ forms of selected sugars. Nature, 1961, 191, 810-811.

PaUL, T. Physikalische Chemie der Lebensmittel: V. Der Süssungrade der Süsstoffe. Zeitschrift für Elektrochemie, 1921, 27, 539-546.

STEVENS, J. C. A comparison of ratio scales for the loudness of white noise and the brightness of white light. Unpublished doctoral dissertation, Harvard University, 1957.

STEVENS, S. S. The psychophysics of sensory function. American Scientist, 1960, 48, 226-253.

STEVENS, S. S. Sensory scales of taste intensity. Perception \& Psychophysics, in press.

STEVENS, S. S., \& STEVENS, J. C. Dynamics of visual brightness. Unpublished monograph, Laboratory of Psychophysics, Harvard University, 1960, PPR 246.

\section{NOTES}

1. Research supported in part by a National Science Foundation predoctoral fellowship and in part by National Institute of Health grant to Harvard University (Laboratory of Psychophysics Report PPR-351).

2. Current address: U.S Army Natick Laboratories, Pioneering Research Laboratories, Natick, Mass. 01760.

(Accepted for publication September 30, 1969.) 